\title{
A Fuzzy Recognizing Method of polyhedrons under Affine Transformation
}

\author{
Yanmin $\mathrm{Li}^{1, \text { a }}$, Jun Zhao ${ }^{2, \mathrm{~b}}$ \\ ${ }^{1}$ School of Mechatronic Engineering, Lanzhou Jiaotong University,Lanzhou,730070,China \\ ${ }^{2}$ School of Mechatronic Engineering, Lanzhou Jiaotong University,Lanzhou,730070,China \\ aemail: liyanmin@mail.ıztu.cn, bemail:zhaojun@mail.ıjtu.cn
}

Keywords: affine invariant; triangle areas ratio of the same hemline; polygon; recognition

\begin{abstract}
In this paper, an algorithm is proposed to recognize polygons. As a characteristic invariant the affine invariant triangle areas ratio of the same hemline was used to describe the polygon, and the fuzzy theory was applied in its recognition. Firstly, a pair of edges of two polygons - a target polygon and a model polygon for matching beginning was identified by a reinforced local property. Then, the degrees of membership between each pair of edges of two polygons were calculated by a normal school membership function. Lastly, the final result was obtained based on threshold principle and maximum membership principle. The experiment results show that the algorithm is efficient, robust and not susceptible to noise.
\end{abstract}

\section{Introduction}

In computer vision, pattern recognition, computational geometry, geographic information systems and many other fields, the recognition of polyhedrons is a fundamental problem. Most surfaces of the objects have polyhedrons, and many image recognition have been matched by the contour of polyhedrons, so it is of great significance and meaningfulness to recognize polyhedrons in various positions.

Lots of scholars have already done many studies for the recognition of polyhedrons under affine transformation. Some people use topological affine invariance to identify polyhedrons. For example, Lowe[1]tried to recognize objects according to the parallelism and collinearity of straight line and $\mathrm{Gu}[2]$ did according to the number of vertices and concavity and convexity of polyhedrons. The method of using topological characteristics to recognize polyhedrons is unable to achieve quantitative description, and often cannot accurately recognize and distinguish similar shapes. Others use a variety of affine invariants to recognize, such as a variety of Moment invariants[3] and Fourier descriptors[4]are regarded as the target characteristics to recognize, but these methods generally have the defects of massive calculations. Others use cross ratio of perspective invariants to recognize under the perspective transformation $[5,6]$.

\section{Use area ratio of the same edges to describe polyhedron feature}

\subsection{The area ratio of triangles with the same edges}

Set a quadrilateral ABCD, which affine projection is quadrilateral abcd. In the two quadrilateral, there is two pairs of triangles; one group are triangle ACD and triangle BCD and another are triangle acd and triangle bcd, the former with the edge CD and the latter with the edge cd, as shown in Figure 1. Above affine transformation is expressed as [7]:

$$
\left[x^{\prime} y^{\prime}\right]^{\mathrm{T}}=A[x y]^{\mathrm{T}}+B
$$

About above formula, $A=\left[\begin{array}{ll}a_{11} & a_{12} \\ a_{21} & a_{22}\end{array}\right] ; B=\left[\begin{array}{l}b_{1} \\ b_{2}\end{array}\right]$.

With $S_{\triangle A C D} 、 S_{\triangle B C D} 、 S_{\triangle a c d}$ and $S_{\triangle b c d}$ individually showing the directive area of corresponding triangles, and we can conclude from the formula (1) under affine transformation: 


$$
\left\{\begin{array}{l}
S_{\triangle A C D}=\operatorname{det}\{A\} S_{\triangle a c d} \\
S_{\triangle B C D}=\operatorname{det}\{A\} S_{\triangle b c d}
\end{array}\right.
$$

From above formula (2), two pairs of triangles which have the same edges have the same area ratio, as $S_{\triangle A C D}: S_{\triangle B C D}=S_{\triangle a c d}: S_{\triangle b c d}$. Therefore this is a kind of affine invariant. Otherwise, if the area ratio of triangles which regard a pair of corresponding edges as its edges is equal in two quadrilateral, it can prove that the two quadrilateral are affine correspondence[8].

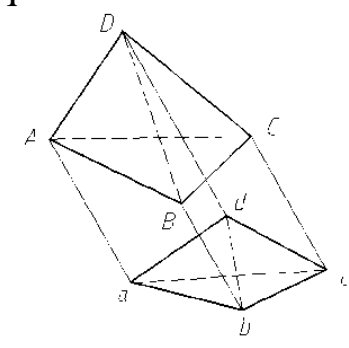

\subsection{Construct characteristic vector of the target model}

Fig. 1. Area ratio of triangles with the same edges

A clockwise polyhedron $P=\left\{e_{1}\left(v_{1} v_{2}\right), e_{2}\left(v_{2} v_{3}\right), \ldots e_{n}\left(v_{n} v_{1}\right)\right\}$ has $N$ edges, with ei representing the ith edge and vi representing the jth vertex, and any edge of $\mathrm{P} e_{i}\left(v_{i} v_{i+1}\right)$ can construct affine invariant (area ratio of triangles with the same edges )with the adjacent two vertices, named Tar $_{i}$ :

$$
\operatorname{Tar}_{i}=\frac{S_{\Delta v_{i-1} v_{i} v_{i+1}}}{S_{\Delta v_{i+2} v_{i} v_{i+1}}}
$$

Provided that the $\mathrm{jth}$ model has $\mathrm{N}_{\mathrm{j}}$ edges in the model library, along the direction of the polyhedron, we can get $N_{j}$ area ratios of triangles with the same edges, and with these invariants arranging sequentially, construct characteristic vector of the model $\mathrm{ChV}_{\mathrm{j}}=\left[\operatorname{Tar}_{\mathrm{j} 1} \operatorname{Tar}_{\mathrm{j} 2} \ldots \operatorname{Tar}_{\mathrm{jNj}}\right]$.

\section{The target type recognition based on fuzzy pattern recognition}

According to the characteristics of issues of the polyhedrons recognition, here we adopt the direct method of fuzzy pattern recognition to solve the matching problem of polyhedrons. Direct method usually has three steps: Choose characteristics of recognition target; Construct membership function under fuzzy pattern and use the principle of maximum membership degree; Judge with the principle of maximum membership degree or the principle of threshold value. After area ratio of triangles with the same edges is determined as characteristic vector, in this section we mainly discuss the following three contents: Establish membership function; Determine the matching start edges of two polyhedrons and judge the target type according to the principle of threshold value and the principle of maximum membership degree.

\subsection{Establish membership function}

$A$,as a fuzzy subset, belongs to discourse domain $U$ refers to: any $\mathrm{u} \in \mathrm{U}$, all specified a number $\mu_{A}(u) \in[0,1]$ as the counterpart, and regard $\mu_{A}(u)$ as membership degree of $u$ relative to $\mathrm{A}$. In fact it constructs a mapping:

$$
\mu_{A}: \begin{aligned}
& U \rightarrow[0,1] \\
& u^{\prime} \rightarrow \mu_{A}(u)
\end{aligned}
$$

$\mu_{A}$ is known as the membership function, and the value of $\mu_{A}(u)$ is called membership degree of $\mathrm{u}$ relative to A. Membership function can be determined based on experience and test by statistical methods , and can also do according to the theoretical analysis. Because the characteristic vector of the target model is a result of an average of large numbers of sample statistics, the distribution is close to normal distribution[9].So membership degree of the sth edge of the recognized target belongs to the $\mathrm{J}$ class model and the th edge is defined as:

$$
M e_{j, t}=\exp \left(-N\left(\operatorname{Tar}_{i}-\mathrm{Tar}_{j t}\right)^{2}\right)
$$

About above formula, $\mathrm{N}$ is the number of edges of the model.

\subsection{Determine starting edge of the matching}

Whether polyhedrons in the model library or to be recognized, the determination of the initial 
edge of them is random. So according to the constructed recognition targets from section 1.2, even if they have the same number of edges, the starting elements of the characteristic vector - area ratio of triangles of the starting edges, generally is not matched. In order to improve the efficiency and accuracy of the recognition, it is necessary to find a pair of closest elements in the characteristic vector of two matching objects, which is to find a pair of most likely matching edges. Use the edges to be a benchmark, and following edges will match in turn. Since two vertices of an edge will affect the characteristic value of the other two adjacent edges, so in order to strengthen local characteristic of the polyhedron on the edge $e_{s}\left(v_{s} v_{s+1}\right)$, use the characteristic value of edge $e_{s}\left(v_{s} v_{s+1}\right)$ and its two adjacent edge $e_{s+1}\left(v_{s+1} v_{s+2}\right)$ and $e_{s-1}\left(v_{s-1} v_{s}\right)$ to form a local characteristic vector.

$$
\text { LPTar }_{s}=\left[\begin{array}{ll}
\left.\operatorname{Tar}_{\mathrm{s}-1}, \operatorname{Tar}_{\mathrm{s}}, \mathrm{Tar}_{\mathrm{s}+1}\right]
\end{array}\right.
$$

So membership degree $M e_{j, t}$ of the start edge characteristic of target to be recognized relative to the th edge of the $\mathrm{j}$ class model in the model library can be obtained:

$M e_{j, t}=\left(M e_{j-1, t-1}+M e_{j, t}+M e_{j+1, t+1}\right) / 3$

Coefficient $1 / 3$ is to ensure the membership degree is between $0 \sim 1$.If $M e_{j},{ }_{k}=\max \left\{M e_{j}\right.$, $\left.{ }_{p}\right\}(p=1,2, \ldots, N)$, the kth edge $e_{k}\left(v_{k} v_{k+1}\right)$ of the $\mathrm{j}$ class model is considered to be the most likely matching edge with the start edge of target to be recognized.

\subsection{Targets Matching}

Determine targets to be recognized and the start edge of the model to be matched ,in the characteristic vector of the model, is to determine the position of eigenvalue of matching with the first eigenvalue in the characteristic vector of targets to be recognized. The rest of the characteristic values of the two vectors sequentially match and calculate membership degree in turn. Such as set the target vector to be recognized as $C h V_{k}=\left[\begin{array}{lll}\operatorname{Tar}_{1} & \operatorname{Tar}_{2} \ldots \operatorname{Tar}_{N}\end{array}\right]_{1 \times N}$, and characteristic vector of the first $\mathrm{j}$ class Model as $\mathrm{ChVj}=\left[\operatorname{Tar}_{\mathrm{j} 1} \operatorname{Tar}_{\mathrm{j} 2} \ldots \operatorname{Tar}_{\mathrm{jN}}\right]_{1 \times \mathrm{N}}$. We assume that the start matching edge is $\operatorname{Tar}_{j i}$ in the determined $C h V_{j}$, then calculate the membership degree by matching $\operatorname{Tar}_{1}$ with $\operatorname{Tar}_{j i}$ in the $\mathrm{ChV}_{\mathrm{k}}$ and sequentially matching $\operatorname{Tar}_{2}$ with $\operatorname{Tar}_{\mathrm{ji}}+1$ until the $\operatorname{Tar}_{\mathrm{N}}$ matches with $\operatorname{Tar}_{\mathrm{ji}}$ 1.Eventually membership degree of characteristic vector of the target to be identified relative to characteristic vector of the first $j$ class model is:

$$
S M e_{j}=\frac{1}{N} \sum_{q=1}^{N} M e_{j, q}
$$

In order to determine the model type of the target to be recognized, we should Combine principle of threshold value with the principle of maximum membership degree. The given threshold value is $\lambda$, and $\operatorname{Max}\left\{\mathrm{SMe}_{1}, \mathrm{SMe}_{2}, \ldots\right\}<\lambda$, which is considered to be that the target does not match any model in the model library, or to judge according to the principle of maximum membership degree. As follows:

$$
S M e_{k}=\max \left\{S M e_{1}, S M e_{2}, \ldots\right\} \geq \lambda
$$

It is considered to be that the target to be recognized matches with the first $\mathrm{k}$ class model in the model library.

Main steps of the algorithm:

Step1: Set each polyhedron of the model in the direction of clockwise; Calculate characteristic vector of the model and establish model library;

Step2: Adjust the polyhedron of target to be recognized in the direction of clockwise and calculate the characteristic vector;

Step3: From the model library use the targets with the same number of vertexes to form a subclass library, and set the first kind of model as the current matching model;

Step4: Determine the matching start edge of the current matching model;

Step5: According to formula (4) calculate the membership degree of each edge of target to be recognized relative to each edge of current model;

Step6: According to Formula (6) calculate the membership degree of the target to be recognized relative to current model;

Step7: In the subclass library take next model as the current model and return to Step4 until go over all models of the subclass library;

Step8: Output model type of matching according to formula (7) and the given threshold value $\lambda$. 


\section{Experimental results}

Test above method in matlab environment, and as shown in Figure 2 bulid the model library of ten polyhedrons; Choose the four targets of figure 3 as the targets to be recognized, and conduct the matching recognition experiment.<smiles>C1CCCCCC1</smiles>

(a)Model

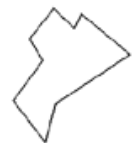

(f)Model VI

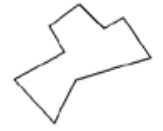

(b)Model

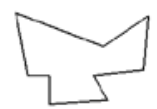

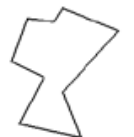

II (c)Model

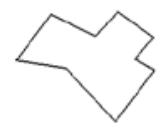

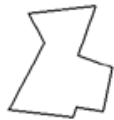

(d)Model

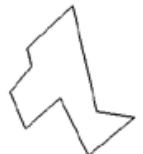

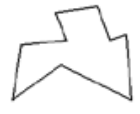

IV (e)Model IV

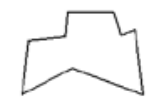

IX (j)Model $\quad X$

Fig. 2. Library model

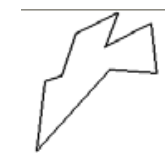

(a)Target1

(g)Model VII

(h)Model VIII (i)Model

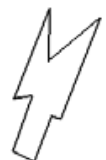

(b)Target 2<smiles>C1CCCCCCCC1</smiles>

(c)Target 3
(d)Target 4

Fig.3. Target to be recognized

The target 1 (FIG. 3 (a)) to be recognized is got through a series of affine transformation by the model $\mathrm{V}$, and random noise under the average $5 \%$ of target coordinate value is attached to each vertex coordinate. From table 1 , its membership degree is $97 \%$ relative to the model V, far more than other membership degrees .The target 2 (FIG. 3 (b)) is the result of parallel projection after the model $\mathrm{X}$ transforms space gesture, and each vertex is added the random noise under average $10 \%$ of the target coordinate value. As we can see from table 2 for model $X$, the membership degree has declined, but still is the tallest of all models and close to $90 \%$.This shows the method in this paper is not sensitive to noise.

Table 1: the membership degrees of target 1 for each edge of ten types of models

\begin{tabular}{|c|c|c|c|c|c|c|c|c|c|c|}
\hline $\begin{array}{c}\text { MAdedel category } \\
\text { Edge number }\end{array}$ & I & II & III & IV & V & VI & VII & VIII & IX & X \\
\hline 1 & 0.0000 & 0.0000 & 0.0420 & 0.0000 & 0.9906 & 0.8718 & 0.8371 & 0.7559 & 0.0000 & 0.0000 \\
\hline 2 & 0.0000 & 0.3972 & 0.0378 & 0.9993 & 0.9878 & 0.9148 & 0.9957 & 0.0000 & 0.0000 & 0.0000 \\
\hline 3 & 0.0000 & 0.0000 & 0.7357 & 0.0000 & 0.9857 & 0.9840 & 0.7509 & 0.0000 & 0.0058 & 0.0000 \\
\hline 4 & 0.0000 & 0.0000 & 0.9845 & 0.0000 & 0.9356 & 0.7406 & 0.9849 & 0.0035 & 0.0000 & 0.0000 \\
\hline 5 & 0.0000 & 0.0000 & 0.5126 & 0.0034 & 0.9327 & 0.9804 & 0.8776 & 0.0000 & 0.0000 & 0.0000 \\
\hline 6 & 0.0218 & 0.0006 & 0.3733 & 0.0000 & 0.9779 & 0.0000 & 0.6669 & 0.0000 & 0.0000 & 0.0183 \\
\hline 7 & 0.0000 & 0.0000 & 0.7337 & 0.0000 & 1.0000 & 0.1732 & 0.6737 & 0.0000 & 0.0000 & 0.0000 \\
\hline 8 & 0.0000 & 0.9825 & 0.6456 & 0.0000 & 0.9651 & 0.4652 & 0.8946 & 0.0006 & 0.3463 & 0.0000 \\
\hline 9 & 0.5757 & 0.0000 & 0.9665 & 0.0086 & 0.9842 & 0.5775 & 0.5124 & 0.0000 & 0.0000 & 0.0282 \\
\hline $\begin{array}{c}\text { Membership } \\
\text { degree SMe } \\
\text { relative to model }\end{array}$ & 0.0664 & 0.1534 & 0.5591 & 0.1124 & 0.9733 & 0.6342 & 0.7993 & 0.0844 & 0.0391 & 0.0052 \\
\hline
\end{tabular}

After the two vertices of model I are changed a lot, we regard it as the target 3 to be recognized, as shown in figure 3 (c). After the matching, the membership degree of its edge for each edge of model I is $0.8531,0.8680,0.9991,0.5593,0.3614,0.7923,0.9317,0.8510,0.9317$.Because the change of a vertex can cause change of adjacent three elements in characteristic vector, the membership degree of its multiple edges and corresponding edge in model I has declined obviously. The membership degree for each type of model is $0.7752,0.1031,0.2161,0.0551,0.1711,0.1879$, $0.1090,0.0338,0.1090,0.0338$.If we set 0.7 as the threshold value $\lambda$, it can still belong to model. If matching precision $\lambda$ is above 0.9 , it can't match with any model. The target 4(as shown in figure 3 (d)) to be recognized is an enneagon taken randomly; After checking the membership degree for each model is $0.3214,0.1668,0.1611,0.0387,0.2723,0.2798,0.2381,0.1381,0.2381,0.1381$. 
Table 2: the membership degrees of target 2 for each edge of ten types of models

\begin{tabular}{|c|c|c|c|c|c|c|c|c|c|c|}
\hline $\begin{array}{c}\text { AMded category } \\
\text { Edge numbek }\end{array}$ & I & II & III & IV & V & VI & VII & VII & IX & X \\
\hline 1 & 0.0000 & 0.3611 & 0.3987 & 0.0166 & 0.4437 & 0.8935 & 0.9508 & 0.1378 & 0.2250 & 0.9803 \\
\hline 2 & 0.0000 & 0.0108 & 0.6955 & 0.0212 & 0.3412 & 0.2668 & 0.9895 & 0.0151 & 0.2929 & 0.9774 \\
\hline 3 & 0.0000 & 0.8437 & 0.9915 & 0.1861 & 0.6250 & 0.8095 & 0.9954 & 0.0319 & 0.0010 & 0.9057 \\
\hline 4 & 0.0000 & 0.0000 & 0.9923 & 0.2459 & 0.7280 & 0.1391 & 0.0938 & 0.7293 & 0.0000 & 0.9816 \\
\hline 5 & 0.1940 & 0.3995 & 0.8849 & 0.7266 & 0.1148 & 0.0020 & 0.2569 & 0.9955 & 0.0000 & 0.4078 \\
\hline 6 & 0.0000 & 0.9580 & 0.8364 & 0.5586 & 0.7815 & 0.3480 & 0.9194 & 0.4607 & 0.6731 & 0.9960 \\
\hline 7 & 0.0000 & 0.6626 & 0.1569 & 0.0000 & 0.9988 & 0.0003 & 0.9961 & 0.1391 & 0.8886 & 0.9944 \\
\hline 8 & 0.0076 & 0.3900 & 0.0804 & 0.1133 & 0.9250 & 0.1680 & 0.7883 & 0.0095 & 0.5372 & 0.8593 \\
\hline 9 & 0.0000 & 0.1479 & 0.5695 & 0.0006 & 0.1897 & 0.0002 & 0.8591 & 0.0052 & 0.5906 & 0.9950 \\
\hline $\begin{array}{c}\text { Membership } \\
\text { degree SMe } \\
\text { relative to model }\end{array}$ & 0.0224 & 0.4193 & 0.6229 & 0.2077 & 0.5720 & 0.2919 & 0.7610 & 0.2805 & 0.3565 & 0.8997 \\
\hline
\end{tabular}

In the test of projections of many different postures of the same target, we set 0.9 as the threshold value $\lambda$, and accuracy of the recognition could get $98 \%$. The reason for misjudgment is mainly deviation of determination of the starting edge. In this paper, area ratio of triangles with the same edges is used to describe characteristics of polyhedrons, and the method accompanies a relatively small number of invariants and calculations and improves the recognition efficiency.

\section{Conclusion}

Area ratio of triangles with the same edges has affine invariance, so it can be used to describe the characteristic of the polyhedrons, and used to recognize the polyhedrons under affine transformation. Using strengthening local information to search for the matching edge of the maximum membership degree and using the matching edge to be a benchmark can solve the problem of inaccurate positions for two elements in the process of matching; The introduction of fuzzy theory effectively reduced the sensitivity of global noise for the target to be recognized, and area ratio of the triangles with the same edges is a local invariant characteristic of polyhedrons. This makes the method can recognize the target effectively under the condition of large deviations with individual vertex. The experimental results show that the method has high accuracy of recognition and small number of calculations. Even under the condition that the model in the model library is very approximate, it can accurately recognize the target. The method can be used to recognize the surface of the target in the engineering drawing and can be approximately viewed as target recognition of remote access to images under affine transformation.

\section{References}

[1] D.G.Lowe. Three-dimensional object recognition from single two-dimensional images[J]. Artificial intelligence, 1987,31(7):355 395.

[2] W.K.Gu,J.Y.Yang and T.S.Huang. Matching perspective views of a polyhedron using circuits[J]. Pattern Analysis and Machine Intelligence,1987,9(3):435 462.

[3] J.Flussr, S.Tomas. Pattern recognition by affined moment invariants[J]. Pattern Recognition, 1993,26(1):167 174.

[4] K.Arbter, WE.Snyder, H.Burkhardt. Application of affined invariant flourier descriptors to recognition of 3D objects[J]. Pattern Analysis and Machine Intelligence, 1990,12(7):640 647.

[5] Guimei Zhang, Mantun Gao, Yunwen Shen. A new method of recognizing polyhedrons under perspective transformation[J]. Machinery Design \& Manufacture, 2007, (2):63 65.

[6] Xiuzhi Zhou, Fang Liu, Runsheng Wang. Recognizing Planar Polygon by Local Invariant Features[J]. Journal of Computer-Aided Design\& Computer Graphics, 2003,15(7):858 862.

[7] Mantun Gao, Shiru Qu, Xiqin Li. Projection theory and method in the study of computer vision[M]. Xian: Northwestern Polytechnical University Press,1998.

[8] J. Flussr. Affine invariants of convex polygons[J]. Image Processing, 2002,11(9):1117 1118.

[9] Shuili Chen, Jinggong Li, Xianggong Wang. Fuzzy set theory and its application [M]. Beijing: Science Press,2005. 